\title{
Genetic Diagnosis and Testing in Clinical Practice
}

\author{
Elizabeth McPherson, MD
}

\begin{abstract}
Genetic testing is defined as "the analysis of human DNA, RNA, chromosomes, proteins and certain metabolites in order to detect heritable disease-related genotypes, mutations, phenotypes or karyotypes for clinical purposes." This article focuses on diagnostic and predictive genetic testing. The latter includes presymptomatic testing, which identifies individuals who are expected to become ill in the future and predisposition testing, which identifies those who are at increased risk of becoming ill. Decisions regarding genetic testing must be based not only on the analytic accuracy, availability and cost of the test, but on the clinical utility as well, including the sensitivity, specificity and interpretability of results. Clinical information, including the medical and family history and the findings of the physical examination, is vital for the selection of appropriate diagnostic tests, as well as the interpretation of the results. Presymptomatic genetic testing is a very personal choice that should only be made after the patient has had sufficient counseling to develop an understanding of the risks and benefits of the test and is able to make an informed decision. The same principle applies to predisposition testing; however, additional factors, such as the probability of a positive result, the likelihood that the disease will actually develop in those with positive results, the effect on the management of the index patient, the effects on family members, the risk of false reassurance if the result is negative or the potential for loss of hope if it is positive, all contribute to the assessment of risk versus benefit. Clinical evaluation and counseling of the patient who is at risk for a genetic disorder are labor intensive but essential for the selection and interpretation of genetic tests.
\end{abstract}

Keywords: Apo E; BRCA; Charcot-Marie-Tooth; DNA; Factor V Leiden; Genetic testing; Hereditary hemochromatosis; Huntington's disease; Marfan syndrome; Predictive testing

$\mathrm{W}$

In order to talk about genetic diagnoses and testing, we first need to agree upon what is meant by genetic testing. Genetic diagnoses do not always depend on karyotype or DNA studies. They can be made by a variety of means, including the physical examination, family history, routine hematology, chemistry or pathology studies, and radiological and electrophysiological examinations. For example, an insurance company that does not cover the cost of genetic tests cannot decline payment for a physical examination because it happened to reveal skin lesions diagnostic for neurofibromatosis. Similarly, an institutional review board that requires informed consent for genetic testing certainly cannot require informed consent for a chest X-ray that shows situs inversus and bronchiectasis leading to a diagnosis of Kartagener syndrome. These examples were chosen because

Reprint Requests: Dr. Elizabeth McPherson, Medical Genetics Services, Marshfield Clinic, 1000 North Oak Avenue, Marshfield,WI 54449, Tel: 7I 5-22 I-7400; Fax: 7I5-389-4399; E-mail: mcpherson.elizabeth@marshfieldclinic.org they illustrate how standard medical care can lead to definitive genetic diagnoses.

Obviously, the concept of genetic testing cannot include all of medicine. Genetic testing must focus on those tests that are unique to genetics. With the rapid proliferation of both tests and known diseases, concepts of genetic testing are changing. Initially, genetic testing was primarily biochemical. Since 1959, when LeJeune discovered the chromosomal cause of Down syndrome, rapid advances in cytogenetics have led to new methods of genetic testing, such as banding and later fluorescence in situ hybridization (FISH), and to the discovery of many new chromosomal conditions.

In the 1970s, advances in molecular genetics, including restriction enzymes and cloning of human genes, were the impetus for human DNA studies that culminated in the 
Human Genome Project. Over the past 20 years, the number of genetic disorders for which DNA testing is available has increased from about 10 to over 1,000, and the test methods have changed from reliance on linkage to DNA sequencing for recognition of mutations as small as a single nucleotide. With new disciplines such as molecular cytogenetics, the distinction between biochemical, chromosomal and DNA test methods is becoming blurred. The variety of genetic diseases and available genetic tests presents a challenge to the full-time geneticist and is virtually incomprehensible to the average practitioner.

In 1999, the Task Force on Genetic Testing defined a genetic test as:

the analysis of human DNA, RNA, chromosomes, proteins, and certain metabolites in order to detect heritable disease-related genotypes, mutations, phenotypes, or karyotypes for clinical purposes. Such purposes include predicting risk of disease, identifying carriers, establishing prenatal and clinical diagnosis or prognosis. Prenatal, newborn, and carrier screening, as well as testing in high risk families, are included. 1,2

Although the physical examination, family history, and radiological and electrophysiological examinations appear to be excluded even when they lead to the diagnosis of genetic conditions, this definition is still extremely broad and encompasses many tests that are commonly ordered by non-geneticists (e.g., $\alpha_{1}$-antitrypsin in an emphysema patient, hemoglobin electrophoresis to rule out thalassemia trait). At the same time, the task force definition excludes paternity testing as it does not detect disease and also excludes research studies because they are not intended for clinical purposes. Most recent concerns about genetic testing have focused on DNA-based tests because of their novelty and rapid proliferation, the complexity of their interpretation, the sensitive nature of the information they reveal (e.g., paternity, risk to offspring, future disease in a currently healthy person) and their costs.

\section{CLASSIFICATION OF GENETIC TESTING}

Genetic tests can be classified according to their purpose. ${ }^{1}$ The most obvious is diagnostic testing in which a DNA-based test is used to confirm or rule out a specific genetic disorder. Testing for Fragile- $\mathrm{X}$ in a boy with mental retardation is an example of diagnostic genetic testing. The second, and perhaps most controversial, type of genetic testing is predictive testing. This includes presymptomatic and predisposition testing.

In presymptomatic genetic testing, a healthy person is tested for a condition with delayed onset. A positive result indicates that the patient will develop the condition but does not indicate when this will occur. Evaluating a healthy person with a family history of Huntington's disease is an example of presymptomatic genetic testing. While there is no cure for this disease, a positive result can be used for life planning, including reproductive planning, as well as treatment.

Predisposition genetic testing differs from presymptomatic testing in that it informs individuals of an increased or decreased risk of developing the condition in question; however, the degree of certainty is unknown. This most often applies to cancer predisposition testing in which a positive result indicates a need for increased surveillance, while a negative result implies a risk similar to the general population but is not negligible. Eventually, this area could be expanded to include risk estimates for a wide range of common disorders, susceptibilities to environmental risk factors and responses to drugs and other treatments. ${ }^{3}$

A third type of genetic testing is intended to help couples make reproductive decisions. This testing includes carrier testing, prenatal diagnostic testing and pre-implantation testing performed in conjunction with in vitro fertilization. It is very important to understand that reproductive genetic testing is not necessarily tied to abortion. When a family decides to initiate or continue a pregnancy at high-risk for a genetic condition, the information can be used for future planning, such as lifesaving treatment of the infant at birth. Other types of genetic testing not discussed in detail here include screening for newborns and for those in specific ethnic groups, as well as identity testing for paternity, zygosity and forensic purposes.

\section{GENETIC TESTING: PANACEA OR PANDORA'S BOX?}

In an ideal situation, DNA testing is less invasive, less expensive and more accurate than other test methods. A particularly striking example concerns myotonic dystrophy which, prior to DNA testing, was diagnosed by electromyography demonstrating myotonia. This test was expensive, painful and not entirely accurate since it failed to distinguish between myotonic dystrophy and other less severe myotonias and also failed to detect severe congenital cases that present with hypotonia rather than myotonia. Methods, such as muscle biopsy and creatine kinase measurement, used for the diagnosis of other muscular dystrophies are frequently inconclusive when applied to myotonic dystrophy. In the 1990s, the discovery that myotonic dystrophy results from increased repetitions of a DNA triplet on chromosome 19 led to a relatively inexpensive, non-invasive, definitive means of diagnosing myotonic dystrophy at all levels of severity ranging from subclinical to severe congenital forms of the disease.

Despite spectacular success for some diseases such as myotonic dystrophy, DNA testing is still far from becoming a universal gold standard. The reasons for caution regarding DNA testing are similar to the concerns of other laboratory tests: 1 
- Sensitivity: Although genetic testing studies the genome directly, the sensitivity is not necessarily high. Heterogeneity (i.e., the concept that more than one gene can cause a given disease) and the location of promoters or other gene-controlling elements outside the portion of the gene that is tested are the most common reasons that DNA tests fail to identify affected individuals.

- Specificity: A diagnosis is not always made by the presence of a DNA change. Some gene changes are harmless variants, and mutations in a single gene can sometimes cause several different diseases.

- Interpretation: The interpretation of many genetic tests can be complex because 1) the effect of a given mutation may be modified by other genes and the environment, 2) different changes in a given gene may have different results, 3) gray zone or intermediate alleles may cause disease in only a fraction of cases, 4) other genes, the environment and individual factors such as age and gender can affect penetrance so that two individuals with the exact same gene change may have entirely different clinical presentations, and 5) a person with a "disease causing mutation" may appear unaffected. Unfortunately, many laboratories provide only limited help to practitioners in interpreting test results.

- Cost and availability: Genetic testing is labor intensive, and laboratories may not be able to recover the costs of developing tests for rare genetic disorders. Some tests for more common disorders that do have the potential for profit have been patented. The net result is that most DNA tests are expensive and are performed by only a few laboratories. Furthermore, some tests are available only on a research basis.

The marketing of genetic tests is intensive. Not only geneticists but all practitioners and, in some instances, even the public are exposed to advertising for a wide variety of genetic tests. Laboratories tend to emphasize the number of tests available, but the practitioner may have to look elsewhere for information on test sensitivity and disease frequency. Hereditary peripheral neuropathy, also called Charcot-Marie-Tooth (CMT) disease, provides an instructive example. ${ }^{4}$ From a genetic viewpoint, CMT is extremely complex. There are four major types that are, theoretically, distinguishable clinically or by family history. In reality, however, clinical features of CMT often overlap, and the family history may fail to provide clear evidence for any specific pattern of inheritance. Each type of CMT is divided into multiple subtypes that are recognized by a separate genetic mutation. Thus, genetic testing is important to confirm the diagnosis and to establish the pattern of inheritance.

A well-known genetic laboratory offers a "complete CMT panel" for patients with an unknown type of CMT. The informed practitioner will recognize that this panel is not truly complete, because it includes tests for only 9 of the 20 genes known to cause CMT. This understanding is vital for interpretation, because negative results on this panel do not rule out CMT. Furthermore, the tests included in the panel appear to have been chosen for their technical feasibility rather than their clinical utility. A single type, CMT1A, accounts for $40 \%$ of all CMT and is detectable by a relatively simple test that has $98 \%$ sensitivity, but the panel also includes several very rare types which account for $<2 \%$ of all CMT and several tests whose specificities are low $(<2 \%)$ or unknown.

The most cost-effective approach for the patient and practitioner is to start with testing for the most common type of the disease, but unfortunately marketing tends to lead the practitioner away from this common sense approach. To further complicate matters, information about available genetic testing abounds on the Internet, especially on support group websites targeted to specific diseases. Patients frequently are aware of this information and request specific genetic tests.

\section{CLINICIAN AND TECHNICIAN INTERACTION}

In order to maximize the benefits of genetic testing, it is essential to target the test to the patient. This requires an interaction between the clinician and the laboratory. The clinician must use all of the clinical information to create a differential diagnosis. Then, both the clinicians and the laboratory researchers need to work together to devise test methods for specific disorders. Lastly, the laboratory must supply an accurate interpretation of the laboratory results based on the empirical data as well as the theoretical sensitivity and specificity of the tests offered. Accurate diagnoses depend on the administration of the correct tests, which will not always be the newest or most complex. Two case vignettes concerning individuals with a "Marfanoid habitus" illustrate this concept.

\section{Marfanoid Habitus: Background}

Marfan syndrome is an autosomal dominant connective tissue disorder characterized by skeletal features (e.g., tall and thin body build, long arms and legs, arachnodactyly, hyperextensible joints, scoliosis and pectus excavatum), ocular features (e.g., ectopia lentis or high myopia) and cardiac features (e.g., aortic dilation or aneurysm and mitral valve prolapse). Several well-known individuals, such as Olympic volleyball star Flo Hyman and playwright Jonathan Larson, have died of dissecting aortic aneurysms due to Marfan syndrome. Early diagnosis is vital to detect cardiac complications before they become life threatening, but there is no single diagnostic test.

Although the majority of families with well-documented Marfan syndrome have mutations in the fibrillin gene on chromosome 15, there are some families with classical features who do not have identifiable mutations. In these families, a second locus is suspected. To further complicate 
matters, there are some patients with less severe disease or other connective tissue disorders who have mutations in fibrillin. Therefore, the diagnosis of Marfan syndrome is based on a system that incorporates clinical features and laboratory data. ${ }^{5}$

A patient must have involvement of three body systems with major involvement in one of them to be diagnosed with Marfan syndrome. Genetic involvement, in the sense of a fibrillin mutation or an affected first-degree relative, counts as major involvement in one system. The majority of affected patients meet the criteria based on clinical features alone or in combination with family history, but fibrillin testing can confirm the diagnosis in some borderline cases and can also be helpful for testing at-risk relatives, especially those with mild or questionable clinical features.

\section{Case I}

A healthy infant of average length is referred for evaluation because of long fingers and a family history of Marfan syndrome. The father meets the criteria for Marfan syndrome because he has tall stature, arachnodactyly, ectopia lentis and dilated aortic root. Fibrillin testing is not necessary to confirm his diagnosis. The baby is at risk because his father is affected, but more clinical information is needed in order to confirm his diagnosis. After a slit lamp examination and echocardiogram reveal subluxed lenses and dilated aortic root, the infant is diagnosed with Marfan syndrome and is followed for possible progression of his ocular or cardiac involvement. In this instance, the echocardiogram and eye examination not only were more effective than fibrillin testing to confirm the diagnosis, but they also provided the necessary baseline clinical evaluation for treatable complications.

\section{Case 2}

A tall, very thin woman has scoliosis and arachnodactyly. She is also mentally delayed. An eye examination shows only strabismus, which is not a feature of Marfan syndrome, and her echocardiogram is normal. Because she has only skeletal involvement, she does not meet the criteria for Marfan syndrome even if she had a fibrillin gene mutation. Therefore, fibrillin testing is not indicated, but the patient still needs a diagnosis. Her cognitive deficiency is not a part of Marfan syndrome but might be a clue to the correct diagnosis. Conditions that can cause a Marfanoid habitus with mental deficiency include homocystinuria, Lujan-Fryns syndrome (unlikely in this patient because it is X-linked) and mosaic trisomy 8.6 Further evaluation shows normal urine amino acids, but a skin biopsy confirms trisomy 8 mosaicism.

These two examples illustrate how the clinician and the laboratory can work together. First, a differential diagnosis is developed. A first round of tests is then performed. If no diagnosis is made, a new differential is created and more tests are ordered. This process can be repeated until a diagnosis is made. It is important to note that clinical findings guide the choice of laboratory tests and that laboratory test results can direct further clinical evaluation. Ultimately, the diagnosis may depend on the clinical features, laboratory results or both.

\section{PRESYMPTOMATIC GENETIC TESTING Example: Huntington's Disease}

Huntington's disease is a progressive neurodegenerative disorder that affects both motor and cognitive function. Onset is variable, but is usually in the $4^{\text {th }}$ or $5^{\text {th }}$ decade of life. Huntington's disease is caused by expansion of a DNA triplet repeat on the short arm of chromosome 4. Before direct DNA testing became available, the diagnosis was usually made clinically after symptoms were advanced and confirmation was not available until autopsy. DNA testing is extremely accurate with a specificity of $98 \%$ for both diagnostic and presymptomatic testing, but it does not predict the age of onset. Normal repeat numbers provide essentially complete reassurance (i.e., sensitivity of 99\%) that an individual will neither develop the disease nor transmit it to future generations. There are some technical issues to consider, including "gray zone" and intermediate repeat numbers such that a few individuals may have very late onset or even remain unaffected but can transmit the disorder to their offspring. However, the major dilemmas regarding presymptomatic testing for Huntington's disease are ethical. The available test is accurate, reasonably priced and can be applied to an individual without the need to test other family members. Physically, it requires nothing more invasive than phlebotomy. Nevertheless, it is highly controversial, primarily because the treatment for Huntington's disease remains palliative.7,8

From the outset, it has been recognized that presymptomatic testing should be the patient's decision. Test results, positive or negative, can provide information that is useful for reproductive and life-planning decisions. For some individuals at risk, ending the uncertainty is paramount, but not everyone wants to know that he or she is destined to develop a fatal disease for which there is no prevention or cure. Potential adverse effects for those found to be affected are easy to imagine and include depression and possibly even suicide; loss of personal relationships; concerns about entering long-term commitments, such as education, marriage or childrearing; fear of passing the condition onto future generations; job discrimination and uninsurability. Even those found to be unaffected may suffer some unanticipated consequences, such as survivor guilt. With these concerns in mind, presymptomatic testing programs have usually involved detailed protocols, including genetic counseling and neurological and psychological evaluations, prior to testing. It is important that the results of presymptomatic testing are given during a face-to-face counseling session with a support person present for the patient.

\section{GENETIC PREDISPOSITION:A FRAMEWORK FOR DECISIONS ABOUT TESTING}

Major factors to consider regarding predisposition testing include:

1. The frequency with which detectable mutations are expected in the group tested

a. Whether or not the group can be refined

b. Whether or not the test can be refined 
2. The frequency with which the disease occurs in the group with detectable mutations

3. The benefits of the test

a. Whether or not the results will alter the clinical management of the index patient

b. Whether or not the results are important in caring for family members

4. The risks of the test
a. Cost
b. False reassurance, if results are negative
c. Hopelessness, if results are positive
d. Psychosocial risks

The outline above is a simplified approach to considering the clinical validity, clinical utility, and the ethical and psychosocial implications of a given test. The answers to the questions raised depend, in turn, upon many factors, including the frequency of the disease in question, the relative risk conferred by the gene in question, and the analytic validity of the test. In different populations with their own gene and disease frequencies, and social and economic structures, the risk-benefit ratios may be entirely different. An excellent review provided by Moore et $\mathrm{al}^{9}$ focused on children, but is applicable to other groups as well. The examples provided below have been chosen to illustrate each of the major issues from the decision-making outline for predisposition testing.

\section{Frequency of Mutations in the Group Tested: Improving Cost-Benefit Ratios by Refining the Group}

\section{Example: Genetic Testing for Breast and Ovarian Cancer}

Breast cancer is very common and affects $7 \%$ of all women by the age of 70 years. This makes breast cancer a potential target for predisposition testing, but at the same time, it poses some challenges. Although about $40 \%$ of breast cancer is familial, only about $10 \%$ can be attributed to recognizable heritable mutations. ${ }^{10}$ Two genes, BRCA1 and BRCA2, account for approximately $85 \%$ of the identifiable genes, and due to patent issues, only a single laboratory in the United States is able to offer BRCA1/2 genetic testing at a cost of $\$ 3000$ per test. This laboratory, which markets the test to all women's health care providers, has developed educational materials for geneticists, other health care providers and patients explaining the test and its possible results. ${ }^{10}$ Nevertheless, the interpretation of the test results is complex. Positive results are usually clinically useful; however, precise recommendations for screening and prophylaxis remain controversial. With a known deleterious mutation, lifetime cancer risks can be very high (i.e., up to $87 \%$ for breast cancer and $44 \%$ for ovarian cancer) but screening, especially for ovarian cancer, is imperfect.

Prophylactic medications are still at the research stage, and even mastectomy or oophorectomy does not prevent all breast or ovarian cancers. Furthermore, up to one-third of all detectable mutations are variants of unknown significance (VUS). In order to determine if a given VUS tracks with the cancer in a family, the above-mentioned laboratory does offer free testing to certain relatives. However, physicians must interpret the test results based on the personal and family history of the patient. Negative results on BRCA genetic tests provide only limited reassurance unless there is a family member with a known mutation, and even then, it is important to recall that $>90 \%$ of breast cancer cases are not due to BRCA mutations. Therefore, patients with negative results still need to follow the population screening guidelines. Even in the most reassuring situation (e.g., a patient with no history of cancer who tests negative for a known deleterious mutation found in her affected relatives), there may be complex counseling issues, such as survivor guilt. Adequate counseling prior to testing and for the interpretation of the results often requires several hours of a trained professional's time.

Clearly, with the cost of BRCA genetic tests and the need for extensive counseling in order to maximize the benefit and minimize the risks, testing of the entire female population or even all breast cancer patients is impractical. Therefore, an algorithm must be devised to identify the patients who are most likely to benefit from genetic testing. ${ }^{11,12}$ Families containing multiple individuals with breast and/or ovarian cancer, individuals with pre-menopausal onset of their tumors and individuals with multiple primaries are the most likely to harbor recognizable genetic changes. Many insurers, including Medicare, have already developed criteria based on the number and age of onset of affected family members. BRCA tests have also been refined with the recent addition of testing for deletions and major rearrangements rather than for just classic mutations. Additional research is also being directed at discovering other genes that contribute to hereditary breast cancer. The laboratory has published extensive educational materials for patients, families and physicians to assist in the appropriate use of the test and the interpretation of the results.

\section{Frequency of Disease When a Mutation is Detected: Minimizing False Positives by Refining the Group}

\section{Example: Venous thromboembolism}

Venous thromboembolism (VTE) is a relatively common event and is usually multifactorial. Common non-genetic risk factors for VTE include immobilization, injury, certain malignancies, surgery, childbirth and, to a lesser extent, oral contraceptives and hormone replacement. Several genetic factors, including factor $\mathrm{V}$ Leiden, factor II (prothrombin) variant, protein $\mathrm{S}$ and protein $\mathrm{C}$ deficiencies, and thermolabile variant of methylene tetrahydrofolate reductase, also contribute to thrombophilia risk. However, unselective genetic testing is impractical due to the large number of false positives. For example, the overall risk of VTE in factor V Leiden heterozygotes is $<1 \%$ per year, which is certainly not enough to justify the risk or expense of providing long-term prophylactic medication to the $8 \%$ of the population who have factor $\mathrm{V}$ Leiden. Instead, those with factor $\mathrm{V}$ Leiden must be 
checked for other risk factors, genetic and environmental, to determine if prophylaxis is necessary. If the endpoint is VTE, individuals with only a single genetic risk factor (e.g., factor $\mathrm{V}$ Leiden) are clinically false positives who experience considerable unnecessary expense and anxiety but ultimately do not require treatment. True positives with high genetic risk for VTE are persons with multiple genetic risk factors for thrombophilia. To identify individuals who require prophylaxis, either continuously or at times of surgery or other stress, the ideal test strategy is to target individuals who have early onset or multiple VTEs but only minimal environmental risk factors. The American College of Medical Genetics consensus statement on factor V Leiden mutation testing ${ }^{13}$ recommends testing of individuals with early (i.e., before the age of 50 years), unprovoked or multiple VTEs, as well as those with VTEs at unusual sites or with minimal provocation such as childbirth, oral contraceptives or estrogen replacement. They also recommend testing for those who have a strong family history of VTE but do not recommend testing for the general population, healthy women anticipating pregnancy, oral contraceptive users or healthy children. Counseling is essential for those who are tested so that they understand how factor $\mathrm{V}$ Leiden is just one risk factor among many rather than a sinister genetic disease. Some excellent materials are available to the public via the internet. ${ }^{14}$ Primary care providers need to be prepared for questions raised by an increasingly informed public.

\section{Maximizing the Benefits: Choosing Tests to Alter Clinical Management}

Example: Hemochromatosis

Hemochromatosis is a common disorder affecting 1/200 to $1 / 400$ people and is characterized by progressive iron overload. Affected individuals have a high risk of serious complications (e.g., diabetes, liver disease, cardiomyopathy, arthritis) that are completely preventable with phlebotomy. About $90 \%$ of clinically affected patients have mutations in both copies of the HFE gene. Genetic testing for hemochromatosis is relatively simple, because there are only two common disease-causing mutations. ${ }^{15,16}$ Why then has genetic testing for this disease not become routine? The answer is simple - only patients with biochemical evidence of iron overload can benefit from treatment. Therefore, people diagnosed on the basis of genetic testing are followed biochemically until they develop iron overload. Due to other factors, such as diet and blood loss, this only occurs in approximately $40 \%$ to $80 \%$ of individuals with genetic evidence of hemochromatosis. Furthermore, since a few carriers do require treatment, individuals identified as carriers through family studies still require biochemical screening. Skipping the genetic test and going straight to biochemical testing may be an effective means of identifying those in need of treatment. ${ }^{17,18}$ Of course, there are some specific uses for HFE gene testing, such as confirmation of a biochemical diagnosis in a borderline case, identification of carriers for genetic counseling purposes and identification of family members at risk so they can initiate biochemical testing earlier. Counseling prior to DNA testing will help patients and families to understand the benefits and limitations of genetic testing and to make the best choice for themselves and their families.

\section{Risks of DNA Testing for Common Disorders: False Reassurance or Loss of Hope}

\section{Example: Apolipoprotein (Apo) E and coronary artery disease}

We do not need genetic testing to tell us that early heart attacks run in families. Furthermore, many of the known risk factors such as hypertension, diabetes, dyslipidemia and obesity are also familial. Despite extensive research, clinical testing is available for only a few genes that contribute to the risk of coronary artery disease. Because known genes account for only a small percentage of cardiovascular risk, false reassurance is a significant problem. For example, individuals with the ApoE2 allele have a relative risk of 0.76 for heart disease, ${ }^{19}$ but this is certainly not sufficient to justify abandoning reasonable lifestyle precautions such as exercise or a low-fat diet. Furthermore, excessive concern about unfavorable results on the same test can have the unintended result of discouraging lifestyle modifications. People with the ApoE4 allele have a relative risk of 1.5 for coronary artery disease, ${ }^{19}$ which ideally would provide them with advanced warning so they could modify their lifestyle risks. However, some might conclude that if they are destined to have a heart attack anyway, they might as well continue smoking. To add to the potential feeling of hopelessness and loss of personal control, the ApoE4 allele also confers a relative risk of $\geq 2$ for Alzheimer's disease. ${ }^{20}$ Some individuals who become depressed upon learning that they have inherited the "bad" ApoE4 allele might choose not to even try to prevent a heart attack, because they would prefer sudden cardiac death to slowly advancing Alzheimer's disease. On the other hand, since Alzheimer's disease can occur in the absence of the ApoE4 allele, a caregiver might place too much faith in a normal ApoE result and fail to obtain services for a person with obvious clinical features of Alzheimer's disease. ${ }^{21}$ Individuals who are counseled appropriately are more likely to recognize the risks and benefits, decline testing that is not helpful to them, and correctly understand and use results that are helpful.

\section{CONCLUSION}

Genetics is a rapidly advancing area of medicine. Over the past 50 years, new methods such as biochemical, chromosomal and, most recently, DNA-based tests have resulted in an exponential increase in the number of disorders for which genetic testing is available. Molecular genetics, however, is never going to replace clinical medicine. The history and physical examination are essential for the establishment of a differential diagnosis, which is then used as a guide for the selection of relevant genetic tests, interpretation of genetic test results, and planning of prophylaxis or therapy. Rapport with the patient is crucial in explaining the reasons for testing, the test results, the treatment plan and the implications for other family members. 
The standard of care today for patients with genetic disorders requires collaboration among the primary care providers, specialists, laboratories and genetic counselors.

\section{ACKNOWLEDGMENTS}

The author thanks Marshfield Clinic Research Foundation for its support through the assistance of Linda Weis and Alice Stargardt in the preparation of this manuscript for submission.

\section{REFERENCES}

1. Burke W. Genetic testing. N Engl J Med 2002;347:1867-1875.

2. National Association of Health Underwriters. NAHU Position on Genetic Testing. Available at: http://www.nahu.org/government/issues/genetic_discrimination /nahu_position.htm. Accessed October 6, 2005.

3. Khoury MJ. Genetics and genomics in practice: the continuum from genetic disease to genetic information in health and disease. Genet Med 2003;5:261-268.

4. Bird TD. Charcot-Marie-Tooth Hereditary Neuropathy Overview. Available at: http://www.genetests.org/servlet/access?id=8888892\&key=w2FD ECCeO60nU\&gry $=$ INSERTGRY $\& \mathrm{fcn}=\mathrm{y} \& \mathrm{fw}=\mathrm{Q} 5$ if $\&$ filename $=/$ glossary/profiles/cmt/details.html. Accessed October 6, 2005.

5. Dietz HC. Marfan Syndrome. Available at: http://www.genetests.org/servlet/access?id=8888892\&key=K3KnautbOKsI\&gry $=$ INSERTGRY $\& \mathrm{fcn}=\mathrm{y} \& \mathrm{fw}=\mathrm{dn} x K \&$ filename $=/ \mathrm{g}$ lossary/profiles/marfan/details.html. Accessed October 6, 2005.

6. Wisniewska M, Mazurek M. Trisomy 8 mosaicism syndrome. J Appl Genet 2002;43:115-118.

7. Potter NT, Spector EB, Prior TW. Technical standards and guidelines for Huntington disease testing. Genet Med 2004;6:61-65.

8. National Institute of Neurologic Disorders and Stroke. Huntington's disease: hope through research. Available at: http://www.ninds.nih.gov/disorders/huntington/detail_hunting ton.htm. Accessed February 7, 2006.

9. Moore CA, Khoury MJ, Bradley LA. From genetics to genomics: using gene-based medicine to prevent disease and promote health in children. Semin Perinatol 2005;29:135-143.

10. Genetic testing for hereditary cancer syndromes: an educational tool for healthcare professionals. Salt Lake City, Utah: Myriad Genetic Laboratories;2001.

11. American College of Medical Genetics Foundation. Genetic Susceptibility to Breast and Ovarian Cancer: Assessment, Counseling and Testing Guidelines. Available at: http://www.health.state.ny.us/nysdoh/cancer/obcancer/content s.htm. Accessed October 6, 2005.

12. Nelson HD, Huffman LH, Fu R, Harris EL; U.S. Preventive Services Task Force. Genetic risk assessment and BRCA mutation testing for breast and ovarian cancer susceptibility: systematic evidence review for the U.S. Preventive Services Task Force. Ann Intern Med 2005;143: 362-379. Also available at: http://www.ahrq.gov/clinic/uspstf/uspsbrgen.htm. Accessed February 7, 2006.

13. Grody WW, Griffin JH, Taylor AK, Korf BR, Heit JA; ACMG Factor V Leiden Working Group. American College of Medical Genetics consensus statement on factor V Leiden mutation testing. Genet Med 2001;3:139-148.

14. National Human Genome Research Institute. Learning about factor V Leiden thrombophilia. Available at: http://www.genome.gov/15015167. Accessed February 7, 2006.

15. Pietrangelo A. Hereditary hemochromatosis - a new look at an old disease. N Engl J Med 2004;350:2383-2397.
16. Kowdley KV, Tait JF, Bennett RL, Motulsky AG. HFE-Associated Hereditary Hemochromatosis. Available at:

http://www.genetests.org/servlet/access? $\mathrm{db}=$ geneclinics\&site $=\mathrm{gt}$ $\& \mathrm{id}=8888891 \& \mathrm{key}=\mathrm{KJ}$ iFh1qiLqGRp\&gry $=\& \mathrm{fcn}=\mathrm{y} \& \mathrm{fw}=\mathrm{qCD}$ c\&filename $=/$ profiles/hemochromatosis/index.html. Accessed October 6, 2005.

17. Zoller H, Cox TM. Hemochromatosis: genetic testing and clinical practice. Clin Gastroenterol Hepatol 2005;3:945-958.

18. Qaseem A, Aronson M, Fitterman N, Snow V, Weiss KB, Owens DK; Clinical Efficacy Assessment Subcommittee of the American College of Physicians. Screening for hereditary hemochromatosis: a clinical practice guideline from the American College of Physicians. Ann Intern Med 2005;143:517-521.

19. Eichner JE, Dunn ST, Perveen G, Thompson DM, Stewart KE, Stroehla BC. Apolipoprotein E polymorphism and cardiovascular disease: a HuGE review. Am J Epidemiol 2002; 155:487-495.

20. Mayeux R, Saunders AM, Shea S, Mirra S, Evans D, Roses AD, Hyman BT, Crain B, Tang MX, Phelps CH. Utility of the apolipoprotein E genotype in the diagnosis of Alzheimer's disease. Alzheimer's Disease Centers Consortium on Apolipoprotein E and Alzheimer's Disease. N Engl J Med 1998;338:506-511.

21. van der Cammen TJ, Croes EA, Dermaut B, de Jager MC, Cruts M, Van Broeckhoven C, van Duijn CM. Genetic testing has no place as a routine diagnostic test in sporadic and familial cases of Alzheimer's disease. J Am Geriatr Soc 2004;52:2110-2113.

\section{AUTHOR AFFILIATION}

Elizabeth McPherson, MD; Medical Genetics Services, Marshfield Clinic, 1000 N. Oak Avenue, Marshfield, WI 54449 